\title{
Methylenetetrahydrofolate reductase C677T polymorphism is associated with estimated glomerular filtration rate in hypertensive Chinese males
}

\author{
Qing Dong ${ }^{1}$, Genfu Tang ${ }^{2}$, Mingli He ${ }^{3}$, Yunqing Cai ${ }^{1}$, Yefeng Cai ${ }^{4}$, Houxun Xing ${ }^{5}$, Liming Sun ${ }^{6}$, Jianping Li ${ }^{7}$, \\ Yan Zhang ${ }^{7}$, Fangfang Fan ${ }^{7}$, Binyan Wang ${ }^{8}$, Ningling Sun ${ }^{9}$, Lisheng Liu ${ }^{10}$, Xiping X ${ }^{11,12}$, Fanfan Hou ${ }^{12}$, \\ Hongbing Shen ${ }^{1 *}, \mathrm{Xin} \mathrm{Xu}^{12^{*}}$ and Yong Huo ${ }^{7^{*}}$
}

\begin{abstract}
Background: Plasma level of total homocysteine (tHcy) is negatively correlated with kidney function in general population. However, the causal mechanism of this correlation is poorly understood. The purpose of this study is to investigate the association of methylenetetrahydrofolate reductase (MTHFR) C677T gene polymorphism, which is a major genetic determinant of the plasma tHcy level, with estimated glomerular filtration rate (eGFR) in Chinese.

Methods: A total of 18814 hypertensive patients (6 914 males, 11900 females) were included in the study.

Results: Association between the eGFR and MTHFR C677T genotype was examined by sex-specific regression analyses. In males, $\Pi$ genotype was associated with $1.37 \mathrm{ml} / \mathrm{min} / 1.73 \mathrm{~m}^{2}$ decrease in eGFR $(p=0.004)$ and with an increased risk ( $O R=1.32, p=0.008)$ for the lowest quintile of eGFR after adjusting for age, $B M I$, and blood pressures. However, such association was not observed in females ( $p>0.05$ ). This association suggests MTHFR C677T polymorphism may play a role in the regulation of eGFR in males.
\end{abstract}

Conclusions: MTHFR 677 T is a risk allele for decreased kidney function in Chinese males, implicating this gene in the pathogenesis of chronic kidney disease (CKD).

Keywords: MTHFR C677T polymorphism, eGFR, CKD

\section{Background}

Glomerular filtration rate estimated from serum creatinine level (eGFR) is an important measurement of kidney function, and frequently used to define stages of chronic kidney disease (CKD). Plasma level of total homocysteine (tHcy) is negatively correlated with kidney function in general population $[1,2]$. However, the causal mechanism of this correlation is poorly understood. Significant loss of kidney function will inevitably lead to hyperhomocysteinemia, as frequently observed in patients with end-stage renal

\footnotetext{
* Correspondence: hbshen@njmu.edu.cn; xinxxu@gmail.com; huoyong@263. net.cn

${ }^{1}$ Department of Epidemiology and Biostatistics, School of Public Health, Nanjing Medical University, Nanjing, China

${ }^{7}$ Department of Cardiology, Peking University First Hospital, Beijing, China ${ }^{12}$ Institute of Nephrology, Southern Medical University, Guangzhou, China Full list of author information is available at the end of the article
}

disease. On the other hand, hyperhomocysteinemia could induce glomerular injury in animal studies [3-7], though it's not clear if hyperhomocysteinemia play a pathogenic role in decreased kidney function in humans. A recent prospective study suggested tHcy is an independent predictor for CKD [8].

Methylenetetrahydrofolate reductase (MTHFR) catalyzes the conversion of 5,10-methylenetetrahydrofolate to 5methyltetrahydrofolate, a co-substrate for homocysteine remethylation to methionine. C677T, a single nucleotide polymorphism $(C->T)$ at nucleotide position 677 , leads to Ala-> Val codon substitution at amino acid position 222. MTHFR C677T is a major genetic determinant for hyperhomocysteinemia, and has been shown to be associated with risks for stroke, coronary heart disease, neural tube defect, depression, schizophrenia, cancer, and a 
number of other disease statuses [9-14]. However, few have investigated the association between C677T and kidney function. Given the fact that kidney function and tHcy is well correlated and that $C 677 T$ is a major determinant of tHcy, it would be interesting to test if C677T is associated with kidney function in general populations.

In this report, we performed a cross-sectional analysis on association of MTHFR C677T polymorphism with eGFR in 18814 Chinese adults.

\section{Methods}

\section{Study population}

The study subjects were participants of an ongoing China Stroke Primary Prevention Trial (CSPPT, clinicaltrials. gov identifier: NCT00794885) in Lianyungang, Jiangsu province of China. CSPPT is a multi-center randomized controlled trial designed to confirm that enalapril maleate and folic acid tablets is more effective in preventing stroke among the patients with primary hypertension when compared with enalapril maleate. Details for inclusion/ exclusion criteria, treatment assignment, and outcome measures of the trial were described elsewhere (http:// clinicaltrials.gov/ct2/show/NCT00794885). In the current study, we included 18814 subjects from Lianyungang who participated in the screening phase of the trial and had valid measurements of MTHFR C677T genotype and baseline serum creatinine. The Human Subject Committee at the Biomedical Institute of the Anhui Medical University approved the study.

\section{Data collection}

Researchers went to the communities to screen local residents for hypertensive patients. Candidate hypertensive patients were then invited to study centers for a formal screening visit. Each participant was asked to fast after 10 PM the night before the visit. During the screening visit, every participant gave the informed consent and then the following baseline data were collected.

\section{Questionnaires}

Questionnaires were administered to collect information on sociodemographic status, occupation, diet, lifestyle, health behavior, medical history, and medication, as well as reproductive history for women.

\section{Anthropometry}

Height was measured without shoes to the nearest $0.1 \mathrm{~cm}$ on a portable stadiometer. Weight was measured in light indoor clothing without shoes to the nearest $0.1 \mathrm{~kg}$. WC was measured as the minimum circumference between the inferior margin of the ribcage and the crest of the iliac. Hip circumference was measured at the level of maximum extension of the buttocks. BMI was calculated as weight/ height squared $\left(\mathrm{kg} / \mathrm{m}^{2}\right)$.

\section{Blood pressure}

Resting blood pressures were measured three times in a sitting position after 10 minutes of rest using a mercury sphygmomanometer. Three blood pressure reads were then averaged.

\section{Phlebotomy}

Venous blood was drawn from the forearm of each participant in the fasting status. Serum and plasma were separated from blood cells in the field within 30 minutes and kept frozen at $-70^{\circ} \mathrm{C}$.

\section{Laboratory assays and GFR estimation}

Serum creatinine was measured by a modified kinetic rate Jaffe reaction method using a Dade Dimension Chemistry Analyzer (Siemens, Germany). Serum glucose and total cholesterol were also measured on the same analyzer. Plasma homocysteine was measured by an enzyme-cycling method using a Hitachi 7020 Automatic Analyzer (Hitachi, Japan). DNA was extracted from leukocytes in peripheral blood using standard techniques. MTHFR C677T genotype was determined by Taqman assay designed and manufactured by Applied Biosystems (Foster City, CA).

EGFR was estimated using the CKD-EPI equation as following [15]: eGFR $=141 \times \min (\mathrm{Scr} / \mathrm{k}, 1)^{\alpha} \times \max (\mathrm{Scr} /$ $\kappa, 1)^{-1.209} \times 0.993^{\text {Age }} \times 1.018$ [if female], where Scr is serum creatinine $(\mathrm{mg} / \mathrm{dL}), \kappa$ is 0.7 for females and 0.9 for males, $\alpha$ is -0.329 for females and -0.411 for males.

\section{Statistical analyses}

The empirical sex-specific distributions of eGFR were estimated using a kernel density estimating function. Genotype distribution was tested for Hardy-Weinberg equilibrium (HWE) using a $\chi^{2}$ test. Sex-specific regression analyses were performed to investigate the association between eGFR and C677T genotype and to estimate the genotype's odds ratio for low eGFR and CKD, adjusting for age, BMI, and systolic and diastolic pressure. All the analyses were done using the statistical package $\mathrm{R}$ [16].

\section{Results}

This study includes 18814 hypertensive subjects (6914 males, 11900 females) from Lianyungang, China who participated in the screening phase of CSPPT and had valid measurements of baseline serum creatinine level and MTHFR genotype. The phenotypic characteristics of subjects were summarized in Table 1 . In this population females had considerably higher body mass index (BMI) than males ( $26.1 \mathrm{vs} 25.0 \mathrm{~kg} / \mathrm{m}^{2}$ ), while the reverse was true for blood creatinine and tHcy levels. The percentages of subject with diabetic fasting plasma glucose level (FPG $\geq 7.0 \mathrm{mmol} / \mathrm{L}$ ) were $11.7 \%$ and $13.3 \%$, respectively, in males and females. The percentages of subjects with CKD $\left(\mathrm{eGFR}<60 \mathrm{ml} / \mathrm{min} / 1.73 \mathrm{~m}^{2}\right)$ were $3.1 \%$ and $3.4 \%$, 
Table 1 Phenotypic characteristics of study participants

\begin{tabular}{lll}
\hline Variables & Male $(\mathbf{N}=\mathbf{6 9 1 4})$ & Female $(\mathbf{N}=\mathbf{1 1 9 0 0})$ \\
\hline Age, yrs & $60.2 \pm 7.7$ & $59.2 \pm 7.5$ \\
$\mathrm{BMl}, \mathrm{kg} / \mathrm{m}^{2}$ & $25.0 \pm 3.3$ & $26.1 \pm 3.7$ \\
$\mathrm{SBP}, \mathrm{mmHg}$ & $166.4 \pm 20.9$ & $169.1 \pm 20.8$ \\
$\mathrm{DBP}, \mathrm{mmHg}$ & $97.1 \pm 12.3$ & $94.0 \pm 11.5$ \\
Homocysteine, $\mu \mathrm{mol} / \mathrm{L}$ & $11.2(9.3-14.2)$ & $9.4(7.8-11.6)$ \\
$\mathrm{FPG}, \mathrm{mmol} / \mathrm{L}$ & $5.4(4.9-6.2)$ & $5.5(4.9-6.2)$ \\
TC, mmol/L & $5.2(4.5-5.9)$ & $5.4(4.7-6.2)$ \\
Creatinine, mmol/L & $71.0(61.7-81.7)$ & $56.3(48.6-66.0)$ \\
eGFR, ml/min/1.73 m² & $96.4(87.9-104.2)$ & $97.2(87.0-104.5)$ \\
Current smoker, \% & 55.3 & 4.8 \\
MTHFR C677T, \% & & \\
CC & 23.9 & 23.5 \\
CT & 49.3 & 50.2 \\
TT & 26.8 & 26.2 \\
\hline
\end{tabular}

$B M I$ body mass index, SBP systolic blood pressure, DBP diastolic blood pressure, FPG fasting plasma glucose, TC total cholesterol, eGFR estimated glomerular filtration rate, MTHFR methylenetetrahydrofolate reductase. Age, $\mathrm{BMI}$, and blood pressures were given by mean $\pm \mathrm{sd}$, serum markers were given by median (Interquartile range, IQR).

respectively in males and females. The risk-bearing $T$ allele is more common than $C$ allele, with an allele frequency of 0.51 and a $T T$ genotype frequency of 0.26 . The distribution was in Hardy-Weinberg equilibrium ( $p>0.05$ ).

The distributions of eGFR in both genders were quite similar, though it was slightly shifted to the right in female
(Figure 1). Homocysteine and eGFR were inversely correlated, with spearman correlation coefficient rho $=-0.25$ $(-0.22$ in males and -0.28 in females).

Taking eGFR as a quantitative outcome in the regression analyses with adjustment for age, BMI, and systolic and diastolic blood pressure, a significant association of MTHFR C677T genotype and eGFR was observed in males but not in females (Table 2). In males, while there was little difference in eGFR among subjects with $C C$ and $C T$ genotypes, $T T$ homozygotes had $1.37 \mathrm{ml} / \mathrm{min} / 1.73 \mathrm{~m}^{2}$ lower eGFR than $C C$ homozygotes $(\mathrm{p}=0.004)$. Similarly, when dividing subjects in eGFR quintiles and comparing genotype frequencies, higher $T T$ genotype frequencies were observed in the low eGFR quintiles (Q1-3, Table 3) in males. In females, genotype frequencies varied little across eGFR quintiles. In males, $T T$ genotype was associated with a higher risk for decreased kidney function after adjusting for age, BMI, and blood pressures, with OR $=1.32$ (95\% CI:1.08-1.62, $\mathrm{p}=0.008)$ for the lowest quintile of eGFR, $\mathrm{OR}=1.25$ (95\% CI:1.11-1.41, $\mathrm{p}=0.0003$ ) for the lower half of eGFR, and OR=1.21 (95\% CI: 0.89-1.63, $\mathrm{p}=0.22)$ for CKD defined as eGFR $<60 \mathrm{ml} / \mathrm{min} / 1.73 \mathrm{~m}^{2}$. No such association was observed in females.

Since C677T genotype, homocysteine, and eGFR are significantly correlated, it is difficult to segregate the individual effect of genotype and homocysteine on eGFR in a regression analysis. Nevertheless, regression analysis of eGFR with an interactive term between genotype and homocysteine (Table 4) revealed the similar sex-specific

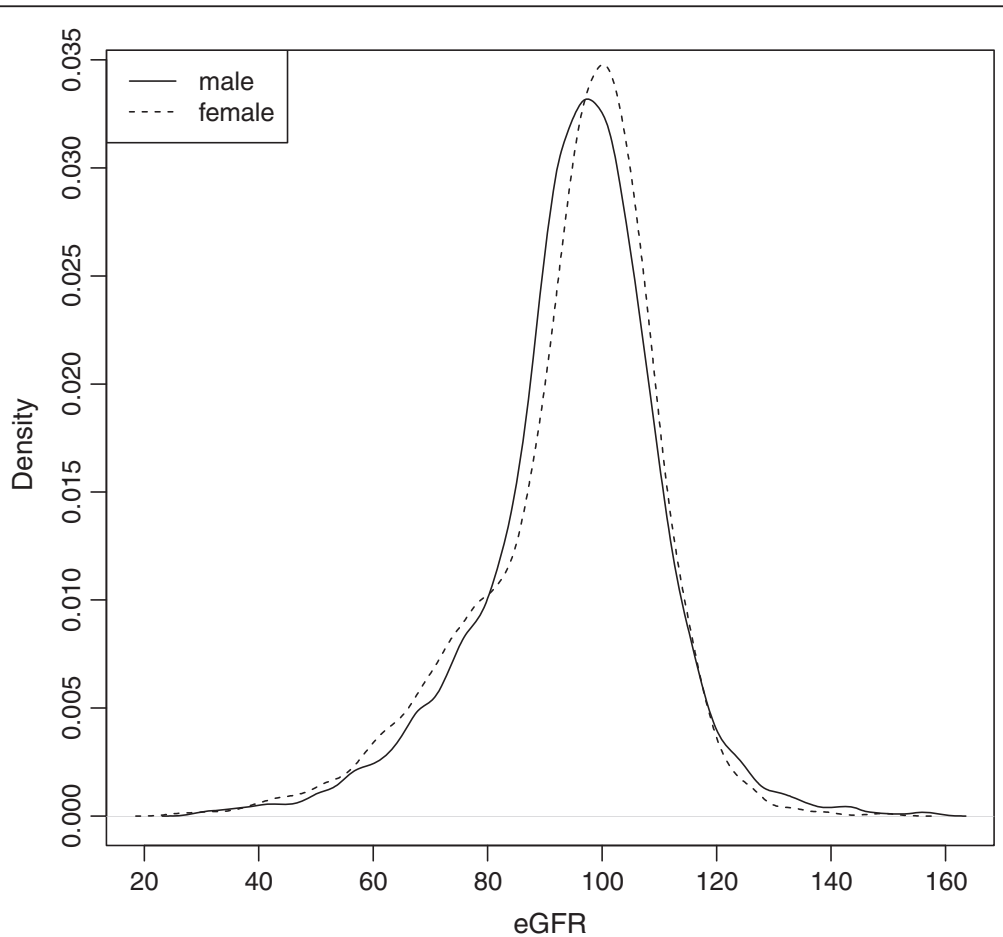

Figure 1 The density curves of eGFR. eGFR = estimated glomerular filtration rate, eGFR was in unit of $\mathrm{ml} / \mathrm{min} / 1.73 \mathrm{~m}^{2}$. 
Table 2 Regression analysis of eGFR and MTHFR C677T genotype

\begin{tabular}{|c|c|c|c|c|c|c|c|c|}
\hline \multirow[t]{2}{*}{ MTHFR C677T genotype } & \multirow[b]{2}{*}{ Mean eGFR } & \multirow[b]{2}{*}{$\beta$} & \multicolumn{2}{|c|}{ Males } & \multirow[b]{2}{*}{ Mean eGFR } & \multirow[b]{2}{*}{$\beta$} & \multicolumn{2}{|c|}{ Females } \\
\hline & & & $S E$ & $P$ & & & $S E$ & $P$ \\
\hline CC & 95.5 & - & & - & 94.7 & - & & - \\
\hline$C T$ & 95.3 & -0.17 & 0.42 & 0.68 & 94.3 & -0.42 & 0.33 & 0.20 \\
\hline$T T$ & 94.1 & -1.37 & 0.47 & 0.004 & 94.7 & -0.19 & 0.37 & 0.61 \\
\hline
\end{tabular}

MTHFR methylenetetrahydrofolate reductase, eGFR estimated glomerular filtration rate, $\beta$ regression coefficient, SE standard error, eGFR was in unit of $\mathrm{ml} / \mathrm{min} /$ $1.73 \mathrm{~m}^{2}$. Regression was done with adjustment for age, BMl, systolic and diastolic blood pressures.

effect of C677T genotype, while plasma homocysteine levels were inversely associated with eGFR in both genders. A significant interaction between TT genotype and homocysteine on eGFR was observed only in males: the regression coefficient of homocysteine was attenuated in TT homozygotes compared with CC homozygotes $(\mathrm{p}=0.002)$.

\section{Discussion}

In this report, we have demonstrated that MTHFR C677T genotype is associated with eGFR in Chinese males, but not in females. The association still holds after adjusting for age, BMI, and blood pressures.

The Val form of MTHFR encoded by the $677 \mathrm{~T}$ allele is thermolabile and has reduced enzymatic activity [9]. The degree of enzyme thermolability (assessed as residual activity after heat inactivation) is much greater in 677TT individuals (18-22\%) compared with C677T (56\%) and C677C (66-67\%). The allele frequency of $677 \mathrm{~T}$ varies with populations, ranging from less than $10 \%$ in African to $50 \%$ in Chinese [12]. Consistent with previous reports, in our study samples $677 T$ is the major allele with an allele frequency of 0.516 and $T T$ homozygote frequency of 0.26 .

Many cross-sectional studies showed that plasma tHcy and kidney function was negatively correlated. For example, in NHANES III study [1], the risk for CKD defined as eGFR $<60 \mathrm{ml} / \mathrm{min} / 1.73 \mathrm{~m}^{2}$ in individuals with high tHcy level ( $>11$ umol/L) was 40 times higher than those with low tHcy level (<7umol/L). However, it's still not clear if hyperhomocysteinemia is the true effector that leads to decreased kidney function or it is simply a marker for kidney function. A recent prospective study in the Framingham cohort demonstrated that baseline homocysteine is an independent risk predictor for CKD and urine microalbuminuria [8]. In animal studies, induced hyperhomocysteinemia could cause podocyte injury and glomerulosclerosis [5-7]. Feeding rats with methionine induced hyperhomocysteinemia and resulted in significant decrease of GFR.

In the current study, we explored association between MTHFR C677T and kidney function indexed by eGFR in a large Chinese sample, and found a significant association in males but not in females. In males, the association model was apparently recessive. While individuals with $C C$ and $C T$ had similar eGFRs, individuals with $T T$ were associated with $1.37 \mathrm{ml} / \mathrm{min} / 1.73 \mathrm{~m}^{2}$ lower value in eGFR, and an increased risk for decreased kidney function defined by bottom 20 or 50 percentiles. Of our study subjects, $3.3 \%$ had CKD with eGFR $<60 \mathrm{ml} / \mathrm{min} / 1.73 \mathrm{~m}^{2}$, which is slightly higher than the $2.53 \%$ prevalence rate in China adults aged 35-74 years reported from the InterAsia [17], partly due to the hypertensive nature of our study sample. Our study also suggested TT genotype was associated with an increase risk of $C K D(O R=1.21, p=0.22)$, though it didn't reach statistical significance probably due to the low percentage of CKD in our sample.

We have also observed a significant interaction between C677T genotype and homocysteine on eGFR in males. In males, the apparent effect size of homocysteine on eGFR was significantly reduced in TT homozygotes compared with subjects with $\mathrm{CC}$ genotype. In other words, the TT effect size was bigger in subjects with lower homocysteine level. However, due to collinearity, it is difficult to segregate their effects under current study design. Another limitation of our study is the study population was hypertensive; the findings from this study may not be generalizable to

Table 3 MTHFR C677T genotype frequencies in eGFR quintiles

\begin{tabular}{|c|c|c|c|c|c|c|c|c|}
\hline \multirow[t]{2}{*}{ eGFR Quintiles } & \multicolumn{4}{|l|}{ Male } & \multirow[b]{2}{*}{$\mathbf{N}$} & \multicolumn{3}{|c|}{ Female } \\
\hline & $\mathrm{N}$ & $C C(\%)$ & $C T(\%)$ & $T T(\%)$ & & $C C(\%)$ & $C T(\%)$ & $T T(\%)$ \\
\hline Q1 (0-20\%) & 2371 & 22.6 & 48.5 & 28.9 & 1375 & 23.6 & 50.6 & 25.8 \\
\hline Q2 (20-40\%) & 2371 & 23.6 & 48.9 & 27.6 & 1375 & 22.9 & 50.4 & 26.7 \\
\hline Q3 (40-60\%) & 2371 & 23.0 & 48.7 & 28.4 & 1375 & 24.0 & 49.1 & 26.9 \\
\hline Q4 (60-80\%) & 2371 & 25.2 & 50.1 & 24.7 & 1375 & 21.9 & 51.3 & 26.7 \\
\hline Q5 (80-100\%) & 2372 & 25.0 & 50.5 & 24.7 & 1375 & 25.3 & 49.7 & 25.0 \\
\hline
\end{tabular}

MTHFR methylenetetrahydrofolate reductase, eGFR estimated glomerular filtration rate. 
Table 4 Regression analysis of eGFR with interaction between homocysteine and MTHFR C677T genotype

\begin{tabular}{|c|c|c|c|c|c|c|}
\hline & \multicolumn{3}{|c|}{ Males } & \multicolumn{3}{|c|}{ Females } \\
\hline & $\beta$ & $S E$ & $P$ & $\beta$ & $S E$ & $P$ \\
\hline$C T$ & -3.54 & 3.12 & 0.26 & -0.83 & 2.30 & 0.72 \\
\hline Tा & -9.39 & 3.16 & 0.003 & -0.79 & 2.48 & 0.75 \\
\hline $\log (\mathrm{Hcy})$ & -6.73 & 1.15 & $5.7 \times 10^{-9}$ & -7.10 & 0.89 & $1.4 \times 10^{-15}$ \\
\hline CT:log(Hcy) & 1.52 & 1.32 & 0.25 & 0.42 & 1.04 & 0.69 \\
\hline TТ:log(Hсy) & 3.91 & 1.29 & 0.0024 & 1.04 & 1.08 & 0.34 \\
\hline
\end{tabular}

Regression was done with adjustment for age, BMI, systolic and diastolic blood pressures (coefficients not shown). $\log (\mathrm{Hcy})$ was the natural log of plasma homocysteine in $\mu \mathrm{mol} / \mathrm{L}$. $\beta$ regression coefficient, SE standard error, eGFR was in unit of $\mathrm{ml} / \mathrm{min} / 1.73 \mathrm{~m}^{2}$.

general population. A confirmation in general population would be helpful.

\section{Conclusions}

In conclusion, we have demonstrated MTHFR $677 T$ is a risk allele for decreased kidney function in hypertensive Chinese males, implicating this gene in the pathogenesis of CKD. Given the relationship among MTHFR, tHcy and eGFR, it would be temptational to speculate that tHcy mediate MTHFR's effect on CKD. The ongoing CSPPT trial, which is essentially a homocysteine-lowering trial, should provide a more definitive answer when it completes.

\section{Abbreviations}

tHcy: Homocysteine; MTHFR: Methylenetetrahydrofolate reductase; eGFR: Estimated glomerular filtration rate; CKD: Chronic kidney disease; BMl: Body mass index; FPG: Fasting plasma glucose level; CSPPT: China Stroke Primary Prevention Trial.

\section{Competing interests}

The authors declare that they have no competing interests.

\section{Authors' contributions}

QD drafted the manuscript. YQC helped to draft the manuscript. XX performed data analysis. XX and FFF edited the manuscript. GFT, MLH, YFC, HXX, LMS, JPL and YZ assisted with data collection, coordination and oversaw sample collection. BYW, NLS, LSL, XPX, FFH, HBS and YH conceived of the study and participated in its design. All authors have read and approved the final manuscript.

\section{Acknowledgement}

This study was supported in part by the Major State Basic Research Development Program of China (973 program) No.2012CB517703.

\footnotetext{
Author details

'Department of Epidemiology and Biostatistics, School of Public Health, Nanjing Medical University, Nanjing, China. ${ }^{2}$ School of Health Administration, Anhui Medical University, Hefei, China. ${ }^{3}$ Department of Neurology, First People's Hospital, Lianyungang, China. ${ }^{4}$ Department of Neurology, Guangdong Traditional Chinese Medicine Hospital, Guangzhou, China. ${ }^{5}$ Lianyungang Center for Advanced Research in Cardiovascular Diseases, Lianyungang, China. ${ }^{6}$ Department of Cardiology, Second People's Hospital, Lianyungang, China. ${ }^{7}$ Department of Cardiology, Peking University First Hospital, Beijing, China. ${ }^{8}$ Institute of Biomedicine, Shenzhen University, Guangzhou, China. 'Department of Cardiology, Peking University People's Hospital, Beijing, China. ${ }^{10}$ Division of Hypertension, Fu-wai Hospital, Beijing, China. "Division of Epidemiology and Biostatistics, University of Illinois at Chicago School of Public Health, Chicago, Illinois 60642, USA. ${ }^{12}$ Institute of Nephrology, Southern Medical University, Guangzhou, China.
}

Received: 14 December 2011 Accepted: 30 April 2012

Published: 16 August 2012

\section{References}

1. Foley RN, Wang C, Collins AJ: Cardiovascular risk factor profiles and kidney function stage in the US general population: the NHANES III study. Mayo Clin Proc 2005, 80:1270-1277.

2. Ruan L, Chen W, Srinivasan SR, Xu J, Toprak A, Berenson GS: Plasma homocysteine is adversely associated with glomerular filtration rate in asymptomatic black and white young adults: the Bogalusa heart study. Eur J Epidemiol 2009, 24:315-319.

3. Hwang SY, Woo CW, Au-Yeung KK, Siow YL, Zhu O K TY: Homocysteine stimulates monocyte chemoattractant protein-1 expression in the kidney via nuclear factor-kappaB activation. Am J Physiol Renal Physiol 2008, 294:F236-F244.

4. Ingram AJ, Krepinsky JC, James L, Austin RC, Tang D, Salapatek AM, Thai K, Scholey JW: Activation of mesangial cell MAPK in response to homocysteine. Kidney Int 2004, 66:733-745.

5. Kumagai H, Katoh S, Hirosawa K, Kimura M, Hishida A, Ikegaya N: Renal tubulointerstitial injury in weanling rats with hyperhomocysteinemia. Kidney Int 2002, 62:1219-1228.

6. Yi F, dos Santos EA, Xia M, Chen QZ, Li PL, Li N: Podocyte injury and glomerulosclerosis in hyperhomocysteinemic rats. Am J Nephrol 2007, 27:262-268.

7. Yi F, Zhang AY, Li N, Muh RW, Fillet M, Renert AF, Li PL: Inhibition of ceramide-redox signaling pathway blocks glomerular injury in hyperhomocysteinemic rats. Kidney Int 2006, 70:88-96.

8. Fox CS, Gona P, Larson MG, Selhub J, Tofler G, Hwang SJ, Meigs JB, Levy D, Wang TJ, Jacques PF, et al: A multi-marker approach to predict incident CKD and microalbuminuria. J Am Soc Nephrol 2010, 21:2143-2149.

9. Frosst P, Blom HJ, Milos R, Goyette P, Sheppard CA, Matthews RG, Boers GJ, den Heijer M, Kluijtmans LA, van den Heuvel LP, et al: A candidate genetic risk factor for vascular disease: a common mutation in methylenetetrahydrofolate reductase. Nat Genet 1995, 10:111-113.

10. Kelly PJ, Rosand J, Kistler JP, Shih VE, Silveira S, Plomaritoglou A, Furie KL: Homocysteine, MTHFR 677 C-> T polymorphism, and risk of ischemic stroke: results of a meta-analysis. Neurology 2002, 59:529-536.

11. Klerk M, Verhoef P, Clarke R, Blom HJ, Kok FJ, Schouten EG: MTHFR 677 C-> T polymorphism and risk of coronary heart disease: a meta-analysis. JAMA 2002, 288:2023-2031.

12. Botto LD, Yang Q: 5,10-Methylenetetrahydrofolate reductase gene variants and congenital anomalies: a HuGE review. Am J Epidemiol 2000, 151:862-877.

13. Muntjewerff JW, Kahn RS, Blom HJ, den Heijer M: Homocysteine, methylenetetrahydrofolate reductase and risk of schizophrenia: a meta-analysis. Mol Psychiatry 2006, 11:143-149.

14. Casas JP, Bautista LE, Smeeth $L$, et al: Homocysteine and stroke: evidence on a causal link from mendelian randomisation. Lancet 2005, 365(9455):224-232

15. Levey AS, Stevens LA, Schmid CH, Zhang YL, Castro AF 3rd, Feldman HI, Kusek JW, Eggers P, Van Lente F, Greene T, Coresh J: A new equation to estimate glomerular filtration rate. Ann Intern Med 2009, 150:604-612. doi:604.

16. R Development Core Team. R: A language and environment for statistical computing. Vienna, Austria: R Foundation for Statistical Computing; 2011. ISBN 3-900051-07-0, URL http://www.R-project.org/. (2011).

17. Casas JP, Bautista LE, Smeeth L, Sharma P, Hingorani AD: Homocysteine and stroke: evidence on a causal link from mendelian randomisation. Lancet 2005, 365:224-232.

doi:10.1186/1471-2350-13-74

Cite this article as: Dong et al:: Methylenetetrahydrofolate reductase C677T polymorphism is associated with estimated glomerular filtration rate in hypertensive Chinese males. BMC Medical Genetics 2012 13:74. 